Tropical Journal of Pharmaceutical Research January 2020; 19 (1): 107-114

ISSN: $1596-5996$ (print); 1596-9827 (electronic)

(C) Pharmacotherapy Group, Faculty of Pharmacy, University of Benin, Benin City, 300001 Nigeria.

\title{
Compound xiebai capsule alleviates pulmonary vascular remodeling in monocrotaline-induced pulmonary arterial hypertension in rats
}

\author{
Zhouye $\mathrm{Wu}^{1,2}$, Zhaoqing $\mathrm{Xi}^{1,2 *}$ \\ ${ }^{1}$ Nanjing University of Chinese Medicine, ${ }^{2}$ Affiliated Hospital of Nanjing University of Chinese Medicine, Nanjing 210029, China \\ *For correspondence: Email: xizhaoqing2019@163.com
}

Sent for review: 18 October 2019

Revised accepted: 29 December 2019

\begin{abstract}
Purpose: To investigate the effect of compound xiebai capsule (CXC) on pulmonary arterial vascular remodeling in a rat model of monocrotaline-mediated pulmonary arterial hypertension (PAH).

Methods: PAH model was established through intraperitoneal injection of MCT (60 mg/kg) to male Sprague-Dawley rats. Thereafter, the rats were grouped and treated with saline (control), nifedipine at a dose of $0.02 \mathrm{~g} / \mathrm{kg} /$ day (positive control), or different doses of CXC $(0.67,1.00$ and $1.32 \mathrm{~g} / \mathrm{kg} /$ day). The effect of treatments on PAH were determined by measurement of hemodynamic indices and right ventricular hypertrophy index (RVHI). Pathological features and ultrastructure of the lung tissue were examined by $H$ \& E staining and transmission electron microscopy (TEM). Immunohistochemistry was employed to determine the expressions of proliferation-associated protein and proliferating cell nuclear antigen (PCNA). Western blotting was applied to assay the expressions of apoptotic pathway proteins.

Results: CXC treatment decreased the levels of hemodynamic parameters in PAH rats, alleviated $R V H I$, improved pulmonary vascular remodeling, decreased vascular wall thickness and area (WT and WA), downregulated the expression of PCNA, and enhanced the expressions of apoptosis-related proteins (cytochrome $C$, caspase-3, and caspase-9).

Conclusion: This is the first study to investigate the effect of CXC on vascular remodeling in PAH. The results indicate that CXC markedly reversed the deleterious changes in PAH-induced rat model.
\end{abstract}

Keywords: Pulmonary arterial hypertension, Pulmonary vascular remodeling, Caspase, Cytochrome C, Apoptosis, Compound xiebai capsule

\begin{abstract}
This is an Open Access article that uses a fund-ing model which does not charge readers or their institutions for access and distributed under the terms of the Creative Commons Attribution License (http://creativecommons.org/licenses/by/4.0) and the Budapest Open Access Initiative (http://www.budapestopenaccessinitiative.org/read), which permit unrestricted use, distribution, and reproduction in any medium, provided the original work is properly credited.

Tropical Journal of Pharmaceutical Research is indexed by Science Citation Index (SciSearch), Scopus, International Pharmaceutical Abstract, Chemical Abstracts, Embase, Index Copernicus, EBSCO, African Index Medicus, JournalSeek, Journal Citation Reports/Science Edition, Directory of Open Access Journals (DOAJ), African Journal Online, Bioline International, Open-J-Gate and Pharmacy Abstracts
\end{abstract}

\section{INTRODUCTION}

Pulmonary arterial hypertension (PAH) is an irreversible disease associated with increased pulmonary blood pressure, reconstructed pulmonary vessels, and poor prognosis. The primary pathology involves continuous increases in pulmonary vascular resistance and increased pulmonary blood pressure. The typical symptoms of PAH include dyspnea, fatigue, angina pectoris and syncope, due to progressive right ventricular failure [1]. Pulmonary hypertension is a very destructive cardiopulmonary disease which, if not treated timely, may lead to deterioration of the patient's condition, resulting in high incidence of right-sided heart failure and premature death [2]. 
A follow-up study of pulmonary hypertension in a multi-ethnic Asian population showed high degree of fatality [3].

Pulmonary arterial hypertension (PAH) is a pathophysiological change in pulmonary vessels caused by different factors. Pulmonary vascular resistance and sustained elevation of pulmonary arterial pressure (PAP) are the main characteristics of pulmonary circulatory diseases which lead to right-sided heart failure [4]. Various humoral factors such as endothelin (ET) and growth factors are involved in this process [5]. The pathological changes include pulmonary vasoconstriction and remodeling, unusual increase in smooth muscles of the lungs and endothelial cells, and in situ thrombosis [6]. The pathological mechanism involved in the development of PAH is complex, but pulmonary vascular remodeling has been identified as the main feature [7].

The pathological basis of pulmonary vascular remodeling is unusual increase in cells of the smooth muscle cells of lung artery and programmed death of the pulmonary artery cells, which at the early stages, increase pulmonary vascular resistance and artery blood pressure [8]. The prevention and treatment of vascular remodeling is an essential strategy in $\mathrm{PAH}$ treatment. Vascular remodeling is a structural and functional change in blood vessels so as to adapt to changes in internal and external environment. Stimulation by external stimuli such as injury and activated smooth muscle cells lead to phenotypic transformation, with significant increase in cell proliferation and migration ability [9]. Vascular remodeling research is primarily focused on inhibition of smooth muscle cell proliferation.

The treatment of PAH with Traditional Chinese Medicine (TCM) has the advantage of focusing on anti-thrombosis, anti-inflammatory response, and anti-vascular remodeling [10]. Compound xiebai capsule (CXC) is a TCM formulation with an empirical formula from the Affiliated Hospital of Nanjing University of Chinese Medicine. It is composed of Allium macrostemon Bunge, Trichosanthes kirilowii Maxim, Pinellia ternata (Thunb.) Breit, Coptis chinensis Franch [11]. It is widely used in the treatment of patients with chronic heart and lung diseases such as chronic obstructive pulmonary disease, chronic bronchitis, severe pneumonia [12-14]. Long-term animal experiments have shown that CXC exerts some pharmacological effects such as reduction of pulmonary artery and right ventricular pressure, protection of pulmonary vascular endothelial cells [15-16]. Previous studies have demonstrated that CXC decreases pulmonary hypertension in rats by modulating plasma endothelin (ET) and interleukin (IL)-6 levels [11]. However, the effect of CXC on pulmonary vascular remodeling in rats with $\mathrm{PAH}$, and the possible underlying mechanisms have not yet been studied. Therefore, this study was carried out to investigate the effect of CXC on a rat model of monocrotaline (MCT)-induced PAH, and the underlying mechanism.

\section{EXPERIMENTAL}

\section{Drugs and reagents}

The drug (CXC) was bought from the Affiliated Hospital of Nanjing University of Chinese Medicine (Batch no. 120301), while MCT was purchased from Sigma-Aldrich (Batch no. WXBC8591V, St. Louis, MO, USA).

\section{Animals and experimental protocols}

Sixty male, SPF SD weighing $200-250 \mathrm{~g}$ (aged 6 - 8 weeks) were used in this study. The animals were kept in a temperature-controlled environment $\left(22-25^{\circ} \mathrm{C}\right)$ with $12 \mathrm{~h}$ light $/ 12 \mathrm{~h}$ dark cycle, and were allowed free access to water and standard laboratory diet. The rats were randomly divided into six groups: normal control, PAH model, positive control, and low-, medium-, and high-dose CXC (CXCL, CXCM, and $\mathrm{CXCH}$, respectively). Rats in normal control group received intraperitoneal injection of physiological saline, while those in other groups received intraperitoneal injections of MCT $(60 \mathrm{mg} / \mathrm{kg})$ for establishment of PAH animal model. The drug was administered from day 2 to day 23. Rats in normal control and PAH model groups were administered saline. Based on a previous study [17], rats in the positive control group were administered nifedipine at a dose of $0.02 \mathrm{~g} / \mathrm{kg}$ per day. Low and high doses of CXC were set as 0.67 and $1.32 \mathrm{~g} / \mathrm{kg}$ per day, respectively [11]. The mean of these doses $(1.0 \mathrm{~g} / \mathrm{kg} /$ day $)$ was taken as the medium dose, which was administered to the CXCM group. The experimental animal protocol used in this study was approved by the experimental animal welfare and ethics committee of Nanjing Agricultural University, China (approval no. P22019026), and performed according to "Principles of Laboratory Animal Care" (NIH publication no. 85 - 23, revised 1985) [18].

\section{Assessment of hemodynamic changes and right ventricular hypertrophy index}

On day 22, after the MCT injections, the rats were anesthetized by intraperitoneal injection of 
$3 \%$ pentobarbital sodium. Then, a catheter filled with $1 \%$ heparin was inserted into the pulmonary artery. The other end of the catheter was connected to biosignal acquisition and analysis system (model BL-420S, Chengdu Techman Software Co., Ltd.) instrument. This experiment served to evaluate mPAP and RVSP. Then, the rats were euthanized, and their hearts were removed. The right ventricle $(R V)$, left ventricle (LV), and septum (S) were excised. After dabbing off the water, the RV and LV + S were weighed. The right ventricular hypertrophy index (RVHI) was expressed as ratio of RV to [LV $+\mathrm{S}]$, as shown in Eq 1.

$\mathrm{RVHI}=\mathrm{RV} /(\mathrm{LV}+\mathrm{S}) \ldots \ldots \ldots(1)$

\section{Hematoxylin and eosin (H\&E) staining}

Rat lung tissue was fixed in $4 \%$ paraformaldehyde for $24 \mathrm{~h}$, and was paraffinembedded after dehydration. Thereafter, it was cut into 5- $\mu \mathrm{m}$ slices which were stained with $\mathrm{H}$ \& $E$, and the structure and shapes of pulmonary vessels were scrutinized using a light microscope (Nikon Eclipse E100, Nikon, Japan). The vascular wall thickness and area percentage (WT and $W A \%$, respectively) were determined as indices of pulmonary vascular reconstruction.

The pathological data were subjected to image analysis with Image-pro Plus 6.0. Pulmonary arterioles of $50 \mu \mathrm{m}<$ diameter $<150 \mu \mathrm{m}$ were selected for each tissue slice, and a x200 magnification scale was used as the standard. The diameter of the external vessel (ED), internal artery (ID), total area of the blood vessel (TA), and area of the blood vessel lumen (IA) were measured so as to calculate WT and WA as shown in Eqs 2 and 3.

$$
\begin{aligned}
& \text { WT }(\%)=[(E D-I D) / E D] \times 100 \ldots \ldots . .(2) \\
& W A(\%)=[(T A-I A) / T A] \times 100 \ldots \ldots . .(3)
\end{aligned}
$$

\section{Immunohistochemistry}

The pulmonary issue was fixed in $4 \%$ paraformaldehyde, embedded in paraffin wax, and the wax blocks were cut into 5- $\mu \mathrm{m}$ sections. The tissue sections were de-waxed in xylene and placed in $0.01 \mathrm{M}$ sodium citrate buffer $(\mathrm{pH} 6.0$ ), followed by heating in a microwave oven at 95 ${ }^{\circ} \mathrm{C}$ for $10 \mathrm{~min}$, and recovery of the antigen. Endogenous peroxidase activity was blocked by incubation in $3 \%$ hydrogen peroxide solution. The tissue was incubated with $3 \%$ bovine serum albumin (BSA) at $25^{\circ} \mathrm{C}$ for $30 \mathrm{~min}$, followed by incubation overnight with anti-proliferating cell nuclear antigen (PCNA) antibody (1:200 dilution;
Servicebio, Wuhan, China) at $4{ }^{\circ} \mathrm{C}$. After washing with phosphate-buffered saline (PBS), the tissue sections were incubated with a goat anti-rat horseradish peroxidase (HRP)-conjugated secondary antibody (1:300 dilution; Servicebio, Wuhan, China) at room temperature for $50 \mathrm{~min}$. Finally, the tissue sections were washed with PBS and stained with 3,3'-diaminobenzidine (DAB, Servicebio, Wuhan, China).

\section{Western blot analysis}

Total protein was extracted from rat lung tissue using radioimmunoprecipitation assay (RIPA) lysis buffer (Beyotime, Shanghai, China). Bicinchoninic acid (BCA) protein assay kit (Beyotime, Shanghai, China) was used to determine the total protein concentration. Then, $20 \mu \mathrm{g}$ protein was subjected to $10 \%$ sodium dodecyl sulfate-polyacrylamide gel electrophoresis (SDS-PAGE) and transferred onto polyvinylidene fluoride membranes $(0.22$ $\mu \mathrm{m})$. The membranes were blocked with $3 \%$ BSA solution for $1 \mathrm{~h}$ at room temperature, and then incubated overnight at $4{ }^{\circ} \mathrm{C}$ with the following primary antibodies (each at 1:1000 dilution): rabbit anti-PCNA, rabbit anti-cytochrome $\mathrm{C}$, rabbit anti-caspase-3 (CASP3), rabbit anticaspase-9(CASP9), and rabbit anti- $\beta$-actin (all from Bioss, Beijing, China).

Thereafter, the membranes were rinsed three times with Tris-buffered saline plus Tween (TBST; 10 min each rinse) and then incubated with goat anti-rabbit IgG/HRP secondary antibody (1:2000 dilution, Bioss, Beijing, China) for $1 \mathrm{~h}$ at room temperature. The membranes were then washed three times with TBST (10 min each rinse), and visualized using enhanced chemiluminescence (ECL, Biosharp, Hefei, China) reagent, and exposed to x-ray film. ImageJ software (National Institutes of Health $[\mathrm{NIH}]$, Bethesda, MD, USA) was used for gray value analysis of the resultant protein bands.

\section{Transmission electron microscopy (TEM)}

Rat lung tissue was cut into $1 \mathrm{~mm} \times 1 \mathrm{~mm} \times 1$ $\mathrm{mm}$ cubes which were fixed for $2 \mathrm{~h}$ at $4{ }^{\circ} \mathrm{C}$ in electron microscopic fixative (G1102, Servicebio, Wuhan, China), followed by treatment with $1 \%$ osmium acid for $2 \mathrm{~h}$ at room temperature. After dehydration in gradient concentrations of acetone, the tissue sections were embedded in resin. Ultra-thin sections $(70-80 \mathrm{~nm})$ were cut, stained with uranium dioxide acetate and lead citrate, and then examined using transmission electron microscopy (TEM, HT7700, Hitachi, Tokyo, Japan) to collect images. 


\section{Statistical analysis}

The experimental results are expressed as mean \pm standard deviation. A one-way analysis of variance (ANOVA) followed by the least significant difference (LSD) or Scheffe post hoc test was used to compare differences among multiple groups. Statistical analyses in this study were performed using the Statistical Package for the Social Sciences (SPSS) software (version 16.0, SPSS Inc., Chicago, IL, USA). Values of $p$ $<0.05$ were considered statistically significant.

\section{RESULTS}

\section{Hemodynamic changes and RVHI}

The values of mPAP $(50.77 \pm 10.83)$ and RVSP $(46.54 \pm 3.49)$ of the MCT group were significantly higher than those of the control group $(p<0.01)$, suggesting that the PAH model was successfully established (Figure $1 \mathrm{~A}$ and $\mathrm{B}$ ). After treatment, the mPAP values of the CXCM and $\mathrm{CXCH}$ groups were significantly decreased, when compared with that of the MCT group $(p<$ $0.01 ; p<0.05$ ), while values of RVSP for all four treatment groups decreased significantly $(p<$ $0.05 ; p<0.01)$. The RVHI increased more significantly in the MCT group than in the control group ( $p<0.01$; Figure $1 \mathrm{C}$ ). However, after treatment, the values decreased significantly in all four treatment groups $(p<0.01)$. These results suggest that CXC lowered the PAP, and improved the hemodynamics changes and right cardiac hypertrophy index of $\mathrm{PAH}$ rats.

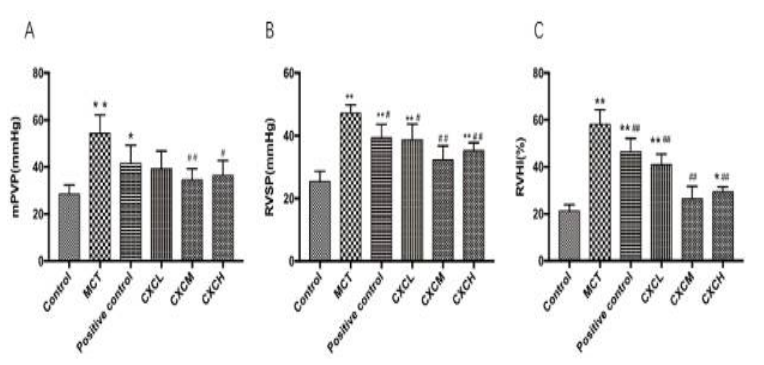

Figure 1: Effect CXC on hemodynamics and RVHI of the PAH rats; ${ }^{*} p<0.05,{ }^{* *} p<0.01$ vs control group; ${ }^{\#} p$ $<0.05,{ }^{\# \#} p<0.01$ vs MCT group

\section{CXC ameliorated pulmonary vascular remodeling in PAH rats}

In the control group, the pulmonary artery vessel walls were thin, the pulmonary tissue structure was clear, and the inflammatory cells in the bronchial and alveolar cavities were few. In the MCT group, the pulmonary artery vessel wall was significantly thicker than that of the control group, with irregular thickening of the vessel wall, narrowing of the lumen, disordered pulmonary tissue structure, extensive infiltration of inflammatory cells, and hyperplasia of pulmonary interstitial collagen fibers. Treatment with different doses of CXC mitigated the $\mathrm{PAH}$ induced vascular remodeling to different degrees: the pulmonary artery vascular wall became thinner than that of the untreated group, inflammatory cell infiltration was reduced, vessel lumen increased, and collagen fiber hyperplasia was reduced (Figure $2 \mathrm{~A}$ ). Pulmonary vascular remodeling was assessed by determining WT and WA, which were significantly higher (31.34 \pm $3.59 \%$ and $47.48 \pm 4.59 \%$, respectively) in the MCT group than in the control group $(p<0.01$, Figure $2 \mathrm{~B}$ ). Furthermore, in the $\mathrm{CXCM}$ and $\mathrm{CXCH}$ groups, values of WA $(27.59 \pm 2.66 \%$ and $28.82 \pm 2.62 \%$, respectively) and WT\% $(12.87 \pm 1.09 \%$ and $13.73 \pm 2.13 \%$, respectively) were significantly lower than those in the MCT group $(p<0.01$, Figure $2 \mathrm{~B})$. These results showed that $\mathrm{CXC}$ mitigated $\mathrm{MCT}$-induced pulmonary vascular remodeling in $\mathrm{PAH}$ rats.

A
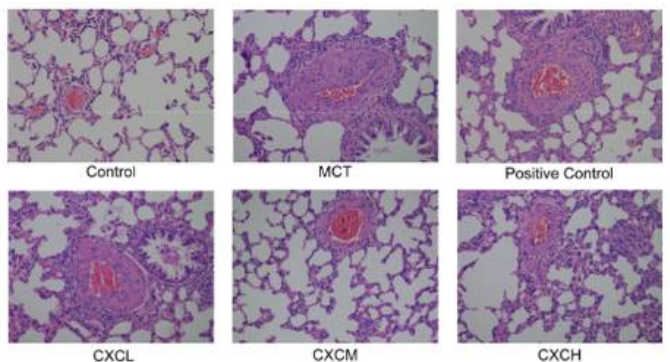

$B$
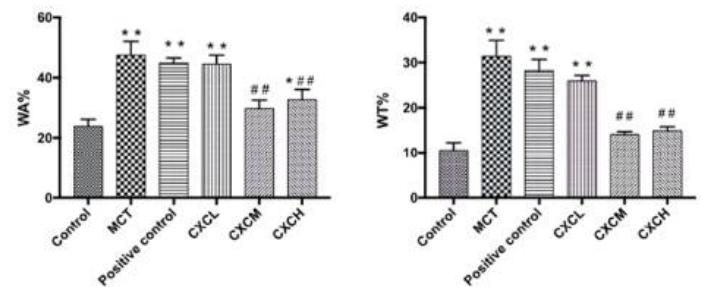

Figure 2: $M C T$-induced pulmonary arterial hypertension $(\mathrm{PAH})$ in rats (scale bar $=100 \mu \mathrm{m})$. $(\mathrm{A})$ Micrographs of rat lung tissues in different groups $(\mathrm{H}$ \& E). (B) Relevant indicators of pulmonary vascular remodeling: percentage vascular wall area (\%WA and percentage vascular wall thickness (\%WT); ${ }^{* *} p<0.01$, ${ }^{*} p<0.05$ vs. control group; $\# \# p<0.01$ vs. MCT group

\section{Immunohistochemical features}

To investigate whether CXC reduced the proliferation of smooth muscle cells, the expression of the proliferation marker PCNA in cells of the lung tissue was detected using immunohistochemistry (Figure $3 \mathrm{~A}$ ). The percentage of PCNA-positive cells in the MCT group $(26.87 \pm 5.32 \%)$ was significantly higher ( $p$ $<0.01$ ) than that in the control group, indicating the presence of proliferation and successful 
establishment of the PAH model. Compared with the MCT group, the percentage of PCNA-positive cells in each treatment group was decreased to different degrees $(p<0.05)$, and the percentage of PCNA-positive cells in the CXCM group was the lowest $(15.45 \pm 2.51 \%)$ of the four treatment groups (Figure $3 \mathrm{~B}$ ). The results showed that CXC reduced pulmonary vascular proliferation, indicating that it ameliorated pulmonary vascular remodeling in MCT-induced $\mathrm{PAH}$ in rats.

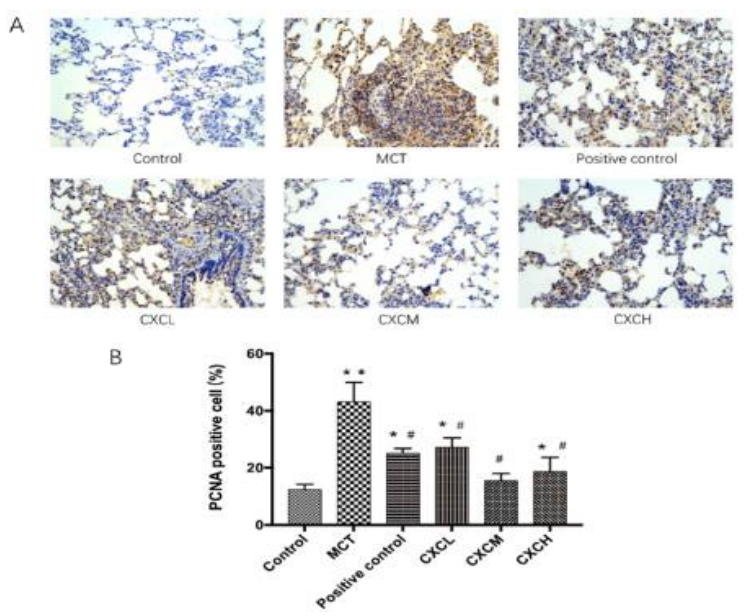

Figure 3: Effect of CXC on pulmonary vascular remodeling of MCT-induced $\mathrm{PAH}$ in rats. The expression of proliferating cell nuclear antigen (PCNA)-positive cells in lung tissue was detected using hematoxylin and eosin (H\&E) staining (scale bar $=100 \mu \mathrm{m}) .{ }^{* *} p<0.01,{ }^{*} p<0.05$ vs control group; $\# p<$ 0.05 vs MCT group.

\section{Effects of CXC on ultrastructure of rat lung tissue}

Ultrastructural changes in pulmonary tissue were determined using TEM (Figure 4). In the control group, the endothelial cells were flat while the structures of the mitochondria, connective tissue, and smooth muscle cells were normal. In the MCT group, the mitochondrial structure was abnormal, and there was vasoconstriction, increased smooth muscle cells, dense collagen fibers, thickened and swollen endothelial cells, narrow capillary lumen, and edema around the capillary. In the positive control group, the smooth muscle cells were relatively reduced, and the mitochondrial structure was abnormal.

In $\mathrm{CXCL}$ group, the mitochondrial structure was restored to normal, collagen fibers were reduced, and endothelial cell swelling was alleviated. In the CXCM group, the mitochondrial structure was improved to some extent, the capillary lumen was relatively normal, and the edema range was small. In the $\mathrm{CXCH}$ group, the mitochondrial structure was relatively abnormal, endothelial cells were swollen and thickened, capillary lumens were relatively narrow, and there were abundant collagen fibers. These results showed that CXC reduced the lung histopathological features of MCT-induced PAH in rats.
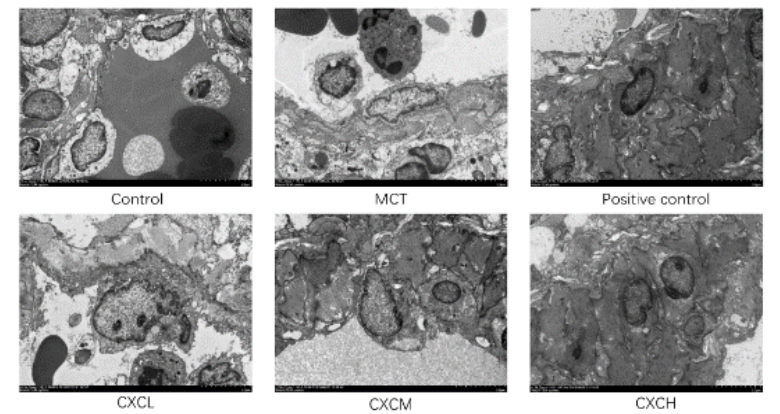

Figure 4: Ultrastructure of rat lung tissue was examined using transmission electron microscopy (TEM, 1500x magnification, scale bar $=5 \mu \mathrm{m}$ ) of structures such as endothelial and smooth muscle cells, mitochondria, and collagen fibers

\section{Western blotting results}

The results of studies on the effect of CXC on the protein levels of proliferation- and apoptosisrelated proteins are shown in Figure $5 \mathrm{~A}$. The expression level of PCNA was significantly higher in the MCT group than in the control group $(p<0.01$, Figure $5 \mathrm{~B})$, whereas the expression levels of cytochrome C, CASP3, and CASP9 were significantly lower $(p<0.01$, Figures 5 C$5 E)$. The CXC treatment significantly decreased PCNA protein levels in all the four groups $(p<$ $0.05 ; p<0.01)$. However, the CXC treatment upregulated protein levels of cytochrome C, CASP3, and CASP9 to different degrees, particularly in the CXCM and $\mathrm{CXCH}$ groups. These results indicate that CXC inhibited the expression levels of PCNA and enhanced those of apoptosis-related pathway proteins i.e. cytochrome C, CASP3, and CASP9.

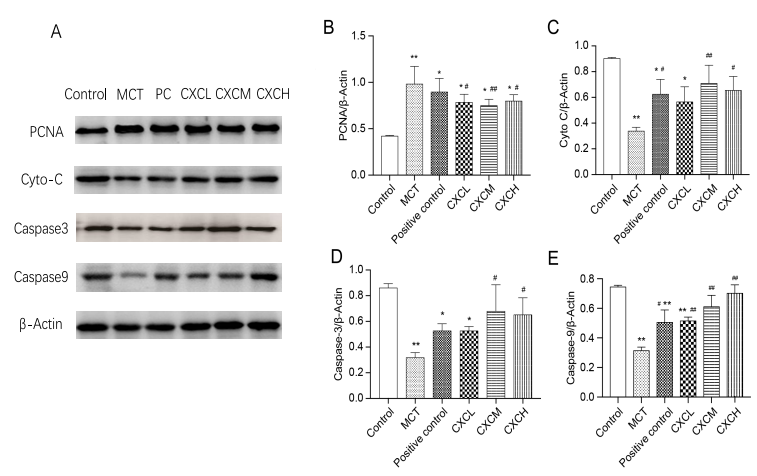

Figure 5: Effect of CXC on expression of PCNA (B), cytochrome $C(C)$, caspase-3 (D), and caspase-9 (E); ${ }^{\star *} p<0.01$ vs control group, $\# p<0.05, \# \# p<0.01$ vs MCT group 


\section{DISCUSSION}

Recently, several studies on pulmonary hypertension demonstrated the advantages of the use of traditional medicines in treating $\mathrm{PAH}$. Previous studies have shown that CXC effectively reduced MCT-induced pulmonary hypertension in rats through mechanisms involving inhibition of the contraction and blockage of proliferation of ET vascular smooth muscles [11]. In this study, the effect of the TCM formulation $\mathrm{CXC}$ on $\mathrm{PAH}$-induced vascular remodeling was investigated. The establishment of $\mathrm{PAH}$ rat model was validated by assessing hemodynamic changes and hypertrophy index. The levels of mPAP, RVSP, and RVHI were determined to evaluate amelioration in right ventricular hypertrophy by CXC. The results showed that levels of mPAP, RVSP, and RVHI in the MCT group were significantly increased, indicating that the model was successfully established. It was observed that treatment with CXC decreased the hemodynamic indices mPAP and RVSP, as well as pulmonary artery pressure in the MCT-treated rats. The decrease in RVHI proved that CXC mitigated right ventricular hypertrophy.

The experimental results from tissue analysis showed increases in wall thickness of pulmonary arterioles of the MCT group, reduction in lumen area, and increases in WT and WA, relative to the normal group. These changes are consistent with pathological changes in pulmonary hypertension, especially the observed enhancement of pulmonary vascular remodeling. Investigations on the effect of some traditional chinese medicine (TCM) interventions in $\mathrm{PAH}$ rats showed reduction in pulmonary vascular WT. These studies evaluated either WT, or described morphological staining images. However, in the present study, WA, WT, and morphological characteristics were more comprehensively and reliably evaluated, and the findings highlight the potential of $\mathrm{CXC}$ as an excellent therapeutic option for $\mathrm{PAH}$.

After treatment, the degree of pulmonary small vessel stenosis and medial thickness of the pulmonary small vessels in the medium- and high-dose groups were significantly reduced, when compared to those in the MCT group. The wall of the pulmonary small vessels became uniformly thinner, the lumen area was larger, and WT and WA were significantly lower than those of the MCT group. These results suggest that CXC may reduce pulmonary artery pressure by reducing the remodeling of pulmonary arterioles and inhibiting the proliferation of smooth muscle cells. However, although CXC showed beneficial effects, it did not completely correct the restructured pulmonary vessels, probably due to the fact that endothelial cell damage caused by pulmonary hypertension is irreversible.

Some studies on the smooth muscle, endothelial cells, organelles, cytoplasm, smooth muscle cells, and connective tissue of the arterial wall have shown that TCM formulations such as tsantan sumtang mitigate $\mathrm{PAH}$-induced ultrastructural changes [19]. The results of the present study agree with those of previous studies on traditional herbal medicines, especially with respect to the CXC-induced amelioration of the deleterious effects of $\mathrm{PAH}$ on pulmonary tissues. Microscopic observation of the ultrastructure of the rat lung tissue showed that the MCT group exhibited abnormal mitochondrial structure, vasoconstriction, increased smooth muscle cells, dense collagen fibers, thickening and swelling of endothelial cells, narrowing of the capillary lumen, and edema around capillaries. These changes were alleviated in the treated groups, with the effects of medium and high CXC doses being more pronounced than that of the low dose. Based on these findings on microcosmic characteristics, CXC partially inhibited and ameliorated $\mathrm{PAH}-$ induced pulmonary artery remodeling in the rat model.

Vascular remodeling in pulmonary hypertension is associated with a variety of factors such as inflammation, endothelial dysfunction, neointimal injury, growth factors, and blood-related factors. It is a dynamic process that generally leads to changes in vessel WT and lumen size [20]. Vascular smooth muscle cells are a rich source of endogenous factors which can be used to promote vascular remodeling through a variety of signal transduction pathways. The accumulation and excessive proliferation of smooth muscle cells result in thickening or even occlusion of the walls of pulmonary arterioles and anterior capillary arteries [21]. Furthermore, these processes narrow the intravascular lumen, thereby increasing resistance to blood flow, which further aggravates the increase in pulmonary artery pressure [21].

The main pathological change in pulmonary hypertension is pulmonary remodeling, a process which is mediated by the proliferation and migration of smooth muscle cells [22]. Therefore, one method used for treating and preventing $\mathrm{PAH}$ is inhibition of proliferation of smooth muscle cells. One of the factors that modulate vascular smooth muscle proliferation is PCNA, which reflects the state of proliferation. To determine whether CXC inhibited the proliferation 
of smooth muscle cells, the expression of PCNA in lung tissue was determined using immunohistochemistry and western blot analyses. The percentage of PCNA-positive cells in each treatment group was smaller (to varying degrees) than that in the MCT group, and the effect produced by CXCM was the most obvious. The protein expression levels supported these conclusions. These results indicate that CXC ameliorated pulmonary vascular remodeling, which may be related to inhibition of cell proliferation. Furthermore, the effect of CXC on apoptosis was studied by assaying the protein expression levels of the apoptosis-related proteins, cytochrome C, caspase-3, and caspase-9 in lung tissue. Previous studies showed that $\mathrm{PAH}$ is associated with endothelial cell apoptosis [23]. However, in the late stage $\mathrm{PAH}$, endothelial cells are resistant to apoptosis and excessive proliferation [24]. Stimulation by various factors cause the release of mitochondrial apoptotic pathway factors (e.g. cytochrome $\mathrm{C}$ ) which activate the downstream caspase family. It is known that CASP9 is a significant promoter of the cytochrome Cdependent reaction cascade, while CASP3, which participates in the transmission of apoptotic signals, is the most crucial executor in the cell apoptosis process [25].

The expression levels of apoptosis-related proteins in the MCT group were decreased, whereas CXC significantly increased the levels of CASP3 and CASP9, which are related to the promotion of the upstream factor cytochrome C. It is likely that CXC initiated or promoted apoptosis since cytochrome C, CASP3, and CASP9 were upregulated. This finding is consistent with those reported in previous studies, where it was shown that CXC inhibited apoptosis in the early stage of $\mathrm{PAH}$, while it promoted apoptosis and reduced proliferation in the late stage [17]. Thus, CXC not only showed inhibitory effects, but it also regulated apoptosis, most likely via a two-way regulatory process. This needs further investigation and validation in subsequent studies.

In the TCM intervention groups, the CXCM and $\mathrm{CXCH}$ doses showed more potent effects than the CXCL, which may be related to increase in drug concentrations. Chinese medicinal formulations are advantageous in the treatment of $\mathrm{PAH}$. The present study provides evidence of a novel potential therapeutic strategy with further research prospects. There are a few limitations of this study. First, it was a preliminary investigation conducted using a rodent model. Thus, translational research to humans is warranted to provide additional mechanistic insights into the various physiological effects of $\mathrm{CXC}$ in PAH. In addition, the precise signal transduction pathway needs to be further elucidated.

\section{CONCLUSION}

To the best of the knowledge of the authors, this study is the first to investigate the effects of CXC on pulmonary vascular remodeling in $\mathrm{PAH}$. It investigated the potential improvement of pulmonary vascular remodeling by CXC in MCTinduced $\mathrm{PAH}$ rats and found that the underlying mechanism of action may be related to the inhibition of smooth muscle cell proliferation and regulation of apoptosis.

\section{DECLARATIONS}

\section{Acknowledgement}

This study was supported by the National Natural Science Foundation of China (No. 81673933).

\section{Conflict of interest}

No conflict of interest is associated with this work.

\section{Contribution of authors}

We declare that this work was done by the author(s) named in this article and all liabilities pertaining to claims relating to the content of this article will be borne by the authors. All authors read and approved the manuscript for publication. Zhao-qing $\mathrm{Xi}$ conceived and designed the study, Zhou-Ye Wu, Zhao-qing Xi collected and analysed the data, while Zhou-Ye Wu wrote the manuscript.

\section{Open Access}

This is an Open Access article that uses a funding model which does not charge readers or their institutions for access and distributed under the terms of the Creative Commons Attribution License (http://creativecommons.org/licenses/by/ 4.0) and the Budapest Open Access Initiative (http://www.budapestopenaccessinitiative.org/rea d), which permit unrestricted use, distribution, and reproduction in any medium, provided the original work is properly credited.

\section{REFERENCES}

1. Galiè N, Humbert $M$, Vachiery JL, Gibbs S, Lang I, Torbicki A, Simonneau G, Peacock A, Vonk 
Noordegraaf A, Beghetti $M$, et al. ESC/ERS Guidelines for the diagnosis and treatment of pulmonary hypertension. Kardiol Pol 2015; 73(12): 1127-1206.

2. Rosenkranz S. Pulmonary hypertension 2015: current definitions, terminology, and novel treatment options. Clin Res Cardiol 2015; 104(3): 197-207.

3. Lim Y, Low TT, Chan SP, Teo TW, Jang JJ, Yip N, Kuntjoro I, Tay EL, Yip JW. Pulmonary arterial hypertension in a multi-ethnic Asian population: Characteristics, survival and mortality predictors from a 14-year follow-up study. Respirol 2019; 24(2): 162-170.

4. Diebold I, Petry A, Djordjevic T, Belaiba S, Fineman JR, Black SM, Schreiber C, Fratz S, Hess J, Kietzmann T, et al. Reciprocal regulation of Racl and PAK-1 by HIF-1 alpha: a positive feedback loop promoting pulmonary vascular remodeling. Antioxid Redox Signal 2010; 13(4): 399-412.

5. Robinson JC, Pugliese SC, Fox DL. Anticoagulation in pulmonary arterial hypertension, Cur Hypertens Rep 2016; 18(6): 47.

6. Chester $A H$, Yacoub $M H$. The role of endothelin-1 in pulmonary arterial hypertension, Glob Cardiol Sci Pract 2014; 2014(2): 62-78.

7. Archer SL, Weir EK, Wilkins MR. Basic science of pulmonary arterial hypertension for clinicians: new concepts and experimental therapies. Circulation 2010; 121(18): 2045-2066.

8. Tuder RM, Archer SL, Dorfmüller P, Erzurum SC, Guignabert C, Michelakis E, Rabinovitch M, Schermuly $R$, Stenmark KR, Morrell NW. Relevant issues in the pathology and pathobiology of pulmonary hypertension. J Am Coll Cardiol 2013; 62(25): 4-12.

9. Sun L, Zhao M, Liu A, Lv M, Zhang J, Li Y, Yang $X, W u$ $Z$. Shear stress induces phenotypic modulation of vascular smooth muscle cells via AMPK/mTOR/ULKImediated autophagy. Cell Mol Neurobiol 2018; 38(2): 541-548.

10. Xiang L, Li Y, Deng X, Kosanovic D, Schermuly RT, Li X. Natural plant products in treatment of pulmonary arterial hypertension. Pulm Circ 2018; 8(3): 2045894018784033.

11. Xi ZQ, Jiang J, Sun JG, Zhu XX, Hong JJ, Xu Y, Gan YM, Feng GK. Inhibitory effect of compound xiebai capsules on pulmonary hypertension induced by monocrotaline in rats. J Tradit Chin Med 2006; (10):781-783.

12. Xu WG, Cui R, Yue CM, Wu JH, Li D, Xie JJ. Association Between 32 -Adrenergic Receptor Gene Polymorphisms and Clinical Outcome in Patients with Chronic Obstructive Pulmonary Disease Treated with Compound Xiebai Capsule. J Hunan Univ Chin Med 2018; 38(12): 1440-1443.
13. Jie C. Clinical effect of compound Xiebai Capsule on patients with acute attack of chronic bronchitis. Clin $J$ Chin Med 2015; 7(25): 83-84.

14. $L u Y, X i Z Q$. Research progress of Baihushen decoction and compound Xiebai Capsule in the treatment of severe pneumonia caused by wind-warming lung fever, Mod J Integr Tradit Chin West Med 2015; 24(35): 39813983.

15. Chen RJ, Wan M, Yan SH, Zhu XX, Rui QL, Xi ZQ. Effect of Compound Macro-stem Onion Capsule on the Expression of VCAM-1 and ICAM-1 in the Lung of Rat models with Pulmonary Hypertension. J Em Tradit Chin Med 2013; 22(8): 1295-1297.

16. Chen RJ, Wan M, Yan SH, Zhu XX, Rui QL, Xi ZQ. Study on the protective mechanism of compound xiebai capsule on pulmonary vascular endothelial cells in rats with pulmonary hypertension at different stages. $\mathrm{J} \mathrm{Em}$ Tradit Chin Med 2014; 23(12): 2207-2211.

17. Xu FY, He CS, Gan JH, Yang DJ. Effects of nifedipine on pulmonary hypertension induced by monocrotaline in rats, Chin J Rehabil Theory Pract 2004; (01):42-43.

18. World Health Organization. Principles of laboratory animal care. WHO Chron 1985; 39: 51-56.

19. He Q, Nan X, Li S, Su S, Ma K, Li Z, Lu D, Ge R. Tsantan sumtang alleviates chronic hypoxia-induced pulmonary hypertension by inhibiting proliferation of pulmonary vascular cells. Biomed Res Int 2018; 2018: 9504158.

20. Renna NF, de Las Heras N, Miatello RM. Pathophysiology of vascular remodeling in hypertension. Int J Hypertens 2013; 2013: 808353.

21. Rabinovitch M. Molecular pathogenesis of pulmonary arterial hypertension. J Clin Invest 2012; 122(12): 43064013.

22. Aggarwal BB, Kunnumakkara AB, Harikumar KB, Gupta $S R$, Tharakan ST, Koca C, Dey S, Sung B. Signal transducer and activator of transcription-3, inflammation, and cancer, Ann N Y Acad Sci 2009; 1171: 59-76.

23. Dieffenbach PB, Maracle M, Tschumperlin DJ, Fredenburgh LE. Mechanobiological Feedback in Pulmonary Vascular Disease. Front Physiol 2018; 9: 951.

24. Lavoie JR, Ormiston ML, Perez-Iratxeta C, Courtman $D W$, Jiang B, Ferrer E, Caruso P, Southwood M, Foster WS, Morrell NW, et al. Proteomic analysis implicates translationally controlled tumor protein as a novel mediator of occlusive vascular remodeling in pulmonary arterial hypertension. Circulation 2014; 129: 2125-2135.

25. Katayama S, Shimoda K, Takenaga Y. Loss of ADAR1 in human iPS cells promotes caspase-3-mediated apoptotic cell death. Genes to Cells 2015; 20(8): 675680. 